\title{
Functional role of brain-engrafted macrophages against brain injuries
}

\author{
Xi Feng 1,2, Elma S. Frias ${ }^{1,2}$, Maria S. Paladini 1,2, David Chen 1,2, Zoe Boosalis ${ }^{1,2}$, McKenna Becker $^{1,2}$, Sonali Gupta ${ }^{1,2}$,
} Sharon Liu ${ }^{3}$, Nalin Gupta ${ }^{3,6,7}$ and Susanna Rosi ${ }^{1,2,3,4,5^{*}}$ (1)

\begin{abstract}
Background: Brain-resident microglia have a distinct origin compared to macrophages in other organs. Under physiological conditions, microglia are maintained by self-renewal from the local pool, independent of hematopoietic progenitors. Pharmacological depletion of microglia during whole-brain radiotherapy prevents synaptic loss and long-term recognition memory deficits. However, the origin or repopulated cells and the mechanisms behind these protective effects are unknown.

Methods: CD45 $5^{\text {low/int }} / C D 11 b^{+}$cells from naïve brains, irradiated brains, PLX5622-treated brains and PLX5622 + whole-brain radiotherapy-treated brains were FACS sorted and sequenced for transcriptomic comparisons. Bone marrow chimeras were used to trace the origin and long-term morphology of repopulated cells after PLX5622 and whole-brain radiotherapy. FACS analyses of intrinsic and exotic synaptic compartments were used to measure phagocytic activities of microglia and repopulated cells. In addition, concussive brain injuries were given to PLX5622 and brain-irradiated mice to study the potential protective functions of repopulated cells after PLX5622 + whole-brain radiotherapy.

Results: After a combination of whole-brain radiotherapy and microglia depletion, repopulated cells are brainengrafted macrophages that originate from circulating monocytes. Comparisons of transcriptomes reveal that brainengrafted macrophages have an intermediate phenotype that resembles both monocytes and embryonic microglia. In addition, brain-engrafted macrophages display reduced phagocytic activity for synaptic compartments compared to microglia from normal brains in response to a secondary concussive brain injury. Importantly, replacement of microglia by brain-engrafted macrophages spare mice from whole-brain radiotherapy-induced long-term cognitive deficits, and prevent concussive injury-induced memory loss.
\end{abstract}

Conclusions: Brain-engrafted macrophages prevent radiation- and concussion-induced brain injuries and cognitive deficits.

Keywords: Microglia, Brain-engrafted macrophages, Cognition, Brain irradiation, CSF-1R inhibitor

\footnotetext{
*Correspondence: Susanna.Rosi@ucsf.edu

${ }^{2}$ Department of Physical Therapy and Rehabilitation Science, University of California San Francisco, Zuckerberg San Francisco General Hospital, 1001 Potrero Ave, Building 1, Room 101, San Francisco, CA 94110, USA

Full list of author information is available at the end of the article
}

\begin{abstract}
Background
Brain-resident microglia are the innate immune cells of the central nervous system (CNS). Arising from the yolk sac during embryonic development, microglia actively survey the environment to maintain normal brain functions $[1,2]$. Under physiological conditions, microglia are maintained solely by self-renewal from the local pool [3]. Following brain injury and other pathological conditions, microglia become activated and play a central role in the
\end{abstract} original author(s) and the source, provide a link to the Creative Commons licence, and indicate if changes were made. The images or other third party material in this article are included in the article's Creative Commons licence, unless indicated otherwise in a credit line to the material. If material is not included in the article's Creative Commons licence and your intended use is not permitted by statutory regulation or exceeds the permitted use, you will need to obtain permission directly from the copyright holder. To view a copy of this licence, visit http://creativecommons.org/licenses/by/4.0/. The Creative Commons Public Domain Dedication waiver (http://creativeco mmons.org/publicdomain/zero/1.0/) applies to the data made available in this article, unless otherwise stated in a credit line to the data. 
clearance of cellular debris, but if not controlled this process can result in aberrant synaptic engulfment [4-7]. Temporary depletion of microglia can be achieved by using pharmacologic inhibitors of the colony-stimulating factor 1 receptor (CSF-1R) [8]. In the normal brain, treatment with CSF-1R inhibitors (CSF-1Ri) can deplete up to 99\% of microglia without causing detectable changes to cognitive functions $[8,9]$. Full repopulation occurs within 14 days of inhibitor withdrawal and the repopulated microglia are morphologically and functionally identical to the microglia in naïve brains [9]. Microglia depletion and repopulation by local progenitors has been shown to be beneficial for disease-, injury-, and age-associated neuropathological and behavioral conditions [10-15]. However, the mechanisms for these protective effects are unknown.

Whole-brain radiotherapy (WBRT), delivered in multiple fractions, is routinely used to treat patients with brain tumors. It is estimated that more than 200,000 patients receive WBRT yearly in the US alone [16]. While it is effective in improving intracranial tumor control, WBRT leads to deterioration of patients' cognitive functions and quality of life [17-19]. Currently, there is no treatment available to prevent or mitigate these adverse effects. Previous studies demonstrated that WBRT causes deleterious effects to the CNS microenvironment by a number of mechanisms including apoptosis of neural progenitor cells, disruption of the blood-brain barrier, activation of microglia and accumulation of peripherally derived macrophages [20-25]. We and others have reported that depletion of microglia during or shortly after brain irradiation in animal models can prevent loss of dendritic spines in hippocampal neurons and cognitive impairments that develop at later time points [12-14]. These reports suggest that microglial plays a critical role in inducing synaptic abnormalities and consequently, cognitive deficits after brain irradiation. The underlying molecular pathways responsible for the protective effects of repopulated microglia against radiotherapy-induced neuronal alterations remain unknown.

In the current study (1), we first defined signature of repopulating cells and analyzed the transcriptional profile of repopulated brain macrophages from irradiated mouse brains after CSF-1R inhibitor-mediated depletion (2). We next sought to establish the origin of repopulated cells coming from the peripheral monocytic lineage (3). We identified the functionality of repopulated macrophages by measuring the ability to engulf synaptic compartments compared to brain resident microglia. Lastly (4), we determined the protective properties of brain-engrafted macrophages (BEMs) against a secondary concussive brain injury-induced cognitive deficits. Together, these results uncover the mechanism by which brain-engrafted macrophages preserve hippocampal synapses and memory functions after radiation injury and also in response to an additional brain injury.

\section{Methods \\ Animals}

All experiments were conducted in compliance with protocols approved by the Institutional Animal Care and Use Committee at the University of California, San Francisco (UCSF), following the National Institutes of Health Guidelines for Animal Care. 7-week-old C57BL/6 J male mice were purchased from the Jackson Laboratory and housed at UCSF animal facilities and were provided with food and water ad libitum. All mice were habituated for one week before any treatments or procedures. 8-10-week-old Cx3cr1 ${ }^{\mathrm{GFP} /+} \mathrm{Ccr} 2^{\mathrm{RFP} /+}$ mice were bred by crossing the $\mathrm{Cx} 3 \mathrm{cr} 1^{\mathrm{GFP} / \mathrm{GFP}} \mathrm{Ccr} 2^{\mathrm{RFP} / \mathrm{RFP}}$ line with wild-type C57BL/6 J mice, and used as donors for the bone marrow chimeras.

\section{CSF-1Ri treatment}

CSF-1Ri (PLX5622 formulated in AIN-76A standard chow at 1200 ppm, Research Diets, Inc.) was provided by Plexxikon, Inc. (Berkeley, CA). Mice were given free access to either CSF-1Ri chow or control diet (AIN-76A without PLX5622) for 21 days. Approximately $4.8 \mathrm{mg}$ of PLX5622 was ingested by each mouse per day in the treated group (calculation based on $4 \mathrm{~g}$ /mouse daily consumption).

\section{Fractionated whole-brain radiotherapy (WBRT)}

8 -week-old mice were injected with ketamine $(90 \mathrm{mg} /$ $\mathrm{kg}) /$ xylazine $(10 \mathrm{mg} / \mathrm{kg})$ mix. When fully immobilized mice were placed in irradiator with cesium-137 source at the dose rate of $2.58 \mathrm{~Gy} / \mathrm{min}$. The body was shielded with a lead collimator that limited the radiation beam to a width of $1 \mathrm{~cm}$ to cover the brain. Three radiation fractions (3.3 Gy) were delivered every other day over 5 days. Sham animals received ketamine/xylazine without irradiation.

\section{Bone marrow chimeras}

8-week-old C57BL/6 J mice were used as bone marrow recipients. 8-week-old males received two doses of 6 Gy cesium-137 irradiation at the dose rate of $2.58 \mathrm{~Gy} /$ min with head protected by lead plates $6 \mathrm{~h}$ apart. Bone marrow cells from 6 to 10 -week-old donors $\mathrm{Cx} 3 \mathrm{cr}^{+1}$ ${ }^{\mathrm{GFP}} \mathrm{Ccr} 2^{+/ \mathrm{RFP}}$ or B6-EGFP (The Jackson Laboratory, stock No 003291) were isolated and resuspended in sterile saline at a concentration of 100 million cells $/ \mathrm{ml} .0 .1 \mathrm{ml}$ of bone marrow cells were injected into recipients via retroorbital injection immediately after the second head-protected irradiation. Bone marrow chimeras were housed 
with $1.1 \mathrm{mg} / \mathrm{ml}$ neomycin as drinking water for 4 weeks and allowed an additional 2 weeks to recover before any treatments.

\section{Concussive TBI—closed head injury}

12-week-old C57BL/6 J mice were randomly assigned to each TBI or sham surgery group. Animals were anesthetized and maintained at $2-2.5 \%$ isoflurane during $\mathrm{CHI}$ or sham surgery. Animals were secured to a stereotaxic frame with nontraumatic ear bars and the head of the animal was supported with foam. Contusion was induced using a 5-mm tip attached to an electromagnetic impactor (Leica) at the following coordinates: anteroposterior, $-1.50 \mathrm{~mm}$ and mediolateral, $0 \mathrm{~mm}$ with respect to bregma. The contusion was produced with an impact depth of $1 \mathrm{~mm}$ from the surface of the skull with a velocity of $5.0 \mathrm{~m} / \mathrm{s}$ sustained for $300 \mathrm{~ms}$. Animals that had a fractured skull after injury were excluded from the study. Sham animals were secured to a stereotaxic frame with nontraumatic ear bars and received the midline skin incision but no impact. After $\mathrm{CHI}$ or sham surgery, the scalp was sutured and the animal was allowed to recover in an incubation chamber set to $37^{\circ} \mathrm{C}$. All animals recovered from the surgical procedures as exhibited by normal behavior and weight maintenance monitored throughout the duration of the experiments.

\section{Synaptosome isolation staining and injection}

Fresh hippocampi from a naïve mouse was homogenized and spun down in $0.32 \mathrm{M}$ sucrose solution (dissolved in $50 \mathrm{mM}$ HEPES buffer). Supernatant was centrifuged in $0.65 \mathrm{M}$ sucrose solution at $12,000 \mathrm{rpm}$ for $30 \mathrm{~min}$ at $4{ }^{\circ} \mathrm{C}$. The synaptosome containing pellet was resuspended in $1 \times$ ice-cold PBS, diluted to $100 \mu \mathrm{g} / \mathrm{ml}$, and stained with PSD-95 antibody (Millipore) on ice for $30 \mathrm{~min}$ followed by a secondary antibody staining (Invitrogen, goat antimouse AF488). Stained synaptosomes were washed and diluted 20 times in PBS and stored at $-80{ }^{\circ} \mathrm{C} .2 \mu \mathrm{l}$ of pre-stained synaptosomes were injected into the right hippocampus of each recipient mouse at the coordinate relative to the bregma: $\mathrm{AP}+1.6 \mathrm{~mm}, \mathrm{ML}+1.6 \mathrm{~mm}$ and DV $-2.0 \mathrm{~mm}$. Mice were euthanized 3 days later. The left hemispheres (uninjected) were used for phagocytosis markers staining and the right hemispheres (injected) were used to assess synaptosome phagocytosis levels by flow cytometry or immunofluorescent staining.

\section{Immunofluorescent staining}

Hemi-brains with synaptosome injection were fixed in $4 \%$ PFA overnight, cryo-protected in $30 \%$ sucrose solution in $1 \times$ PBS and sliced in 20- $\mu \mathrm{m}$ sections. Sliced tissues were stained with Iba1 (Fujifilm Wako Pure Chemical Corporation, 019-19741) followed by a secondary antibody staining (goat anti-rabbit AF568, Invitrogen, A-11011). DAPI was used for nuclear staining. Images close to the injection site (Additional file 1: Fig. S1a) were taken using a Zeiss Imager Z1 microscope under a $20 \times$ objective lens. Tissues from bone marrow chimeras were processed and stained as described above. Images were taken using a CSU-W1 Nikon Spinning Disk Confocal microscope under $10 \times$ air, $20 \times$ air or $100 \times$ immerse oil lenses. All images were analyzed using the Fiji/ImageJ software by experimenters blinded to sample information.

\section{Behavior test}

Novel Object Recognition (NOR) task was used to test hippocampal-dependent recognition memory at 1,3 and 6 months after the last dose of irradiation. All tests took place during the dark cycle in a room with dim red light as previously described $[13,14]$. Briefly, mice were habituated in an open arena $(30 \mathrm{~cm} \times 30 \mathrm{~cm} \times 30 \mathrm{~cm}$, $\mathrm{L} \times \mathrm{W} \times \mathrm{H}$ ) for $10 \mathrm{~min}$ on day one and day two. On day three, two identical objects were put into the arena at a distance of $21 \mathrm{~cm}$ and mice were allowed to explore for 5 min. On day four, one object was replaced by a novel object and mice were allowed to explore for $5 \mathrm{~min}$. All trials were recorded by an overhead camera and analyzed using Ethovision software. Data are presented as discrimination Index, calculated using formula $\mathrm{DI}=\left(\mathrm{T}_{\text {Novel }}-\mathrm{T}_{\text {Familiar }}\right) /\left(\mathrm{T}_{\text {Novel }}+\mathrm{T}_{\text {Familiar }}\right)$.

\section{Flow cytometry}

Mice were perfused with cold PBS after euthanasia. Brains were immediately removed and dissociated using a Neural Tissue Dissociation kit (P) (Miltenyi Biotec). Brain cells were resuspended in 30\% Percoll solution diluted in RPMI medium, and centrifuged at $800 \mathrm{~g}$ for $30 \mathrm{~min}$ at $4{ }^{\circ} \mathrm{C}$. Cell pellets were washed with FACS buffer $(1 \times$ DPBS with $0.5 \%$ BSA fraction $V$ and $2 \%$ FBS $)$, blocked with mouse CD16/32 Fc block (BD Biosciences \#553141) and stained with fluorophore-conjugated antibodies (CD11b-AF700, CD45-FITC, BD Pharmingen 557690 and 553080, C5aR-PE, CD68-PE and CD107aPE, Miltenyi Biotec 130-106-174, 130-102-923 and 130102-219), washed with FACS buffer and used for sort or analyses of bone marrow chimera efficiency. Data were collected on an Aria III sorter using the FACSDIVA software (BD Biosciences, V8.0.1), and analyzed with Flowjo software (FlowJo, LLC, V10.4.2).

\section{Flow synaptometry}

After isolation (described above) synaptosomes were stained with PSD-95 (Abcam ab13552) or Synapsin 1 (Millipore \#1543) antibodies on ice for $30 \mathrm{~min}$, washed and followed by a secondary antibody staining (Invitrogen, goat anti-mouse AF488, A-11001). Stained 
synaptosomes were used immediately for analysis of mean fluorescent intensity measurement. Fluorescent latex beads of $1 \mu \mathrm{m}, 2 \mu \mathrm{m}, 3 \mu \mathrm{m}$ and $6 \mu \mathrm{m}$ were used as references of particle sizes in the FSC-A vs SSC-A dot plot. Events between $1 \mu \mathrm{m}$ and $3 \mu \mathrm{m}$ were used to measure mean fluorescent intensities of isolated synaptosomes under the FITC channel. Data were collected on an Aria III sorter using the FACSDIVA software, and analyzed with Flowjo software. At least 100,000 events were collected from each sample for the analyses.

\section{RNA sequencing}

mRNA was isolated from 100,000 to 400,000 sorted microglia or BEMs $\left(\mathrm{CD} 45^{\text {low/int }} / \mathrm{CD} 11 \mathrm{~b}^{+}\right.$cells) using the Dynabeads mRNA DIRECT Purification Kit (Invitrogen \#61011) following the manufacturer's instructions. RNA sequencing libraries were generated using the Ovation RNA-seq system V2 and Ultralow Library Construction System sample prep kits (NuGEN). Libraries were sequenced on the HiSeq 2500 to generate single end $50 \mathrm{bp}$ reads according to the manufacturer's instructions. Normalized per-gene read counts were used to compare relative gene expression levels across samples. Only genes with average read counts greater than 10 were included for analyses. Heatmaps were drawn using the online analysis software Morpheus (Broad Institute, https://software.broadinstitute.org/morpheus), followed by hierarchical clustering using the One minus Pearson correlation method. Gene Ontology analysis was performed using the Statistical overrepresentation test (GO biological process complete, PANTHER version 14) [26]. Bar graphs to visualize fold enrichment and $p$ values of enriched GO biological pathways were drawn using the GraphPad Prism software (V 7.01, GraphPad Software, Inc.). For analysis of monocyte/microglia signature genes, dataset from Lavin and Winter et al. was used as reference (GSE63340) [27]. Genes significantly up- or down-regulated $(p<0.05$, fold-change $>1.5$ or $<0.667)$ in monocytes vs microglia comparisons are defined as monocyte or microglia signature genes, respectively. Heatmaps were drawn as described above, and similarity matrix was drawn using the Morpheus online tool with Pearson correlation. Monocyte/microglia similarity scores were calculated based on the numbers of genes in each treatment group from this study that expressed in the same trend as monocyte/microglia signature genes (genes with fold-change between 0.6667 and 1.500 or with FDR $>0.01$ were defined as unspecified). For juvenile/embryonic signature analysis, dataset from Matcovitch-Natan and Winter et al. was used as reference (GSE79819) [28]. Gene listed to be highly expressed in yolk sac and embryonic day 10.5-12.5 were defined as embryonic/juvenile microglia signatures, genes highly expressed in adult cortex/hippocampus/spinal cord were defined as adult microglia signatures. Heatmaps, similarity matrix and similarity scores were drawn or calculated as described above.

\section{qPCR}

mRNAs were extracted from sorted microglia using the Dynabeads mRNA DIRECT Purification Kit (Invitrogen \#61011), and reverse transcribed into cDNAs using reverse transcription kit (info). qPCR reactions were set up in duplicate reactions using the PowerUp SYBR Green Master Mix kit (Applied Biosystems \#A25777) using an Mx3000P qPCR System (Agilent, Santa Clara, CA) following the manufacturer's instructions. Data were analyzed using the standard curve method. Standard cDNAs were generated with total RNAs from mixed naïve and irradiated mouse brains. qPCR primers sequences are listed in Additional file 5: Table S4.

\section{Sholl analysis}

Images of GFP + (BEMs from bone marrow chimeras) or Iba1 + (AF555, all microglia cells, and BEMs from nonbone marrow chimeras) cells were acquired from stained frozen Sects. (20 um) using a confocal microscope under $100 \times$ objectives (CSU-W1 Spinning Disk/High Speed Widefield, Nikon). Max Z-projections from Z-stack images (0.26 um step size) were used for Sholl analysis [29] in Fiji [30] software using the following settings: manually defined cell center at the cell body, the numbers of intersections between cellular processes and circles with incremental radius (2 um step size, up to 60um) were recorded, plotted and compared across samples.

\section{Statistical analyses}

Two-way ANOVA was used to determine radiation and CSF-1Ri treatment effects for NOR, qPCR, flow synaptometry, flow cytometry, immunofluorescent staining counts and dendritic spine count results, with Tukey's post hoc multiple comparisons. One-way ANOVA with Sidak's post hoc multiple comparisons was used to determine effect of developmental stages for dataset published by Matcovitch-Natan and Winter et al. Unpaired t-test was used to determine differentially expressed microglia/monocyte signature genes from dataset published by Lavin and Winter et al. Unpaired t-test was used calculate the $p$ value of the comparison of BEMs contributions between the BMT and BMT+WBRT groups. Exact $p$ values and numbers of animals used in each experiment were listed in each related Fig. legend. All error bars represent mean \pm SEM. 


\section{Results}

\section{Microglia depletion and repopulation prevents} radiation-induced hippocampal-dependent memory deficits

Temporary microglia depletion during or shortly after exposure to brain irradiation prevents cognitive deficits, suggesting microglia's key role in modifying neuronal and cognitive functions [12-14]. Changes in expression levels of pro-inflammatory cytokine/ chemokines have been shown to correlate with cognitive performance in mice $[12,15,20]$, however, the exact change in the transcriptional profile of repopulated microglia after brain irradiation is unknown and is an important tool to dissect the roles that repopulated microglia play in preventing of radiation-induced memory deficits. In this study we used a previously reported WBRT and microglia depletion paradigm [13] and performed RNA sequencing using repopulated microglia with and without WBRT, and compared with transcriptomes of microglia obtained from mice without CSF-1R inhibitor-mediated depletion (Fig. 1a, b). A CSF-1R inhibitor was used to fully deplete microglia in 8-week-old male mice, for a duration of 21 days. Three fractions of therapeutic whole-brain irradiation were given to each mouse every other day over five days starting from day 7 of CSF-1R inhibitor treatment. Novel Object Recognition (NOR) test was used to measure recognition memory 4 weeks after the last fraction of WBRT. Consistent with our previous report, fractionated WBRT resulted in impairment in recognition memory, which was prevented by CSF-1R inhibitor treatment (Fig. 1a, lower panel). No deficits in motor functions or changes of anxiety levels were found in the open field test (scored from day 1 of NOR test, data

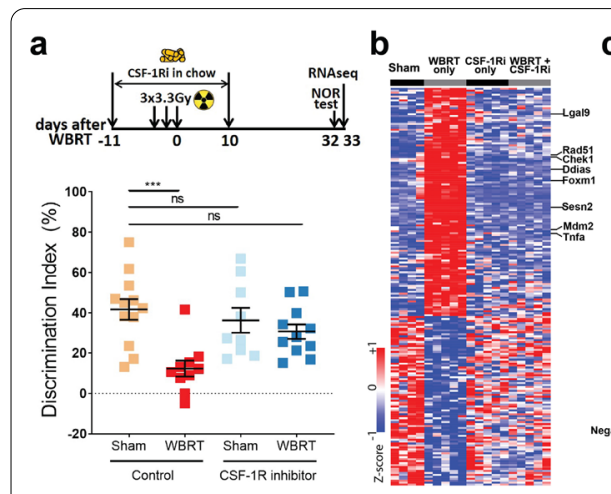

e

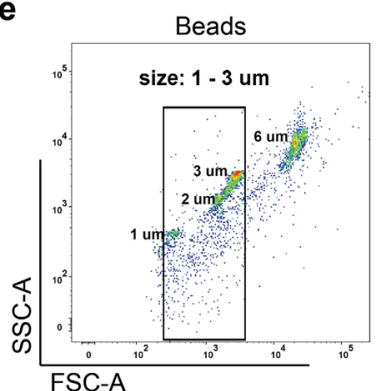

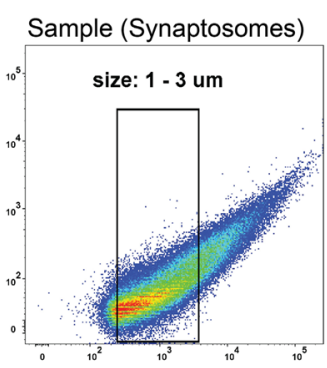

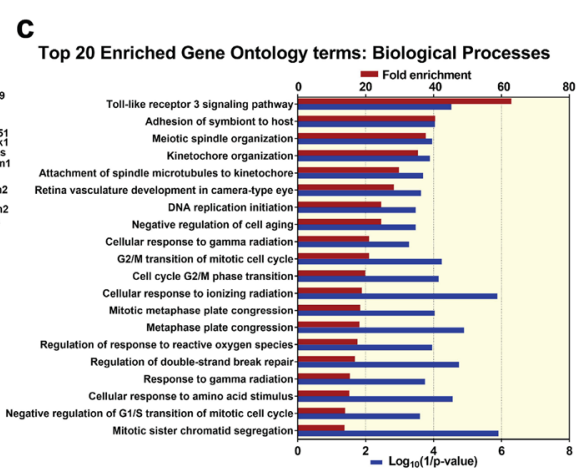

f

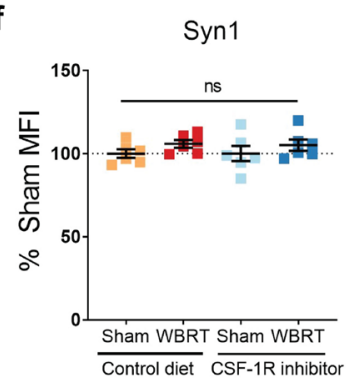

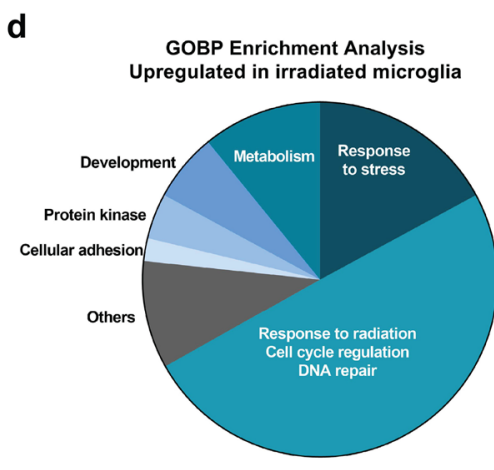

g

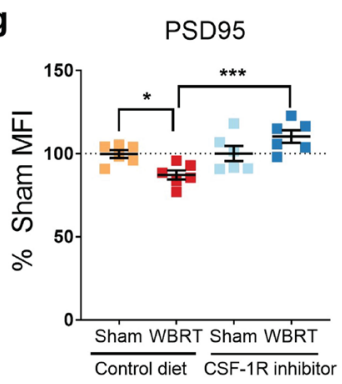

Fig. 1 Microglia depletion and repopulation prevents long-term radiation-induced memory deficits and loss of hippocampal PSD95. a Experimental design and Novel Object Recognition (NOR) test result. CSF-1R inhibitor was used to deplete microglia during 3 doses of 3.3 Gy of whole-brain radiotherapy (WBRT). A 4-day NOR protocol was used to measure recognition memory, which ended on day 32 post WBRT. Microglia were isolated using fluorescent activated cell sorting (FACS) on day 33 and dot plots showing NOR results. Statistical analysis was performed using two-way ANOVA with Dunnett's multiple comparisons test. There is no CSF-1 Ri treatment effect $(F(1,38)=1.787, p=0.1893)$, but significant WBRT effect $(F(1,38)=13.23, p=0.0008)$ and interaction between CSF-1 Ri treatment and WBRT $(F(1,38)=6.07, p=0.0184)$, $N=9-12$, animals with insufficient exploration time on NOR test day were excluded. $\mathbf{b}$ Hierarchically clustered heatmap showing significantly altered microglial genes by WBRT, but not changed with CSF-1Ri treatment. c Bar graphs summarizing fold enrichment and $p$ values of the top 20 enriched Biological Processes by Gene Ontology analysis from up-regulated microglial genes after WBRT (full list in Additional file 2: Table S1). No significantly enriched terms were identified by GO analysis from down-regulated genes by WBRT. $\mathbf{d}$ A pie chart summarizing all enriched GOBP terms. ns = not significant, ${ }^{* * *} p<0.0001$. e Scatter plots showing gating strategy in flow synaptometry analyses. Fluorescent beads at various sizes were used as standard to gate isolated hippocampal cell membrane fractions. Particles between $1 \mu \mathrm{m}$ and $3 \mu \mathrm{m}$ were considered synaptosomes and used to determine Synapsin 1 and PSD95 protein levels by mean fluorescent intensities (MFIs). f Dot plots to compare Synapsin 1 and PSD95 MFI levels in hippocampal cell fractions. Statistical analyses were performed using two-way ANOVA with Tukey's multiple comparisons test. ns $=$ not significant, ${ }^{*} p<0.05$, ${ }^{* * *} p<0.001 . N=6$ 
not shown). One day after the NOR test, mice were euthanized and whole brains were used to sort microglia (Control Sham and Control+WBRT, CD45 $5^{\text {low } /}$ ${ }^{\text {int }} \mathrm{CD} 11 \mathrm{~b}^{+}$) and repopulated cells (CSF-1R inhibitor Sham and CSF-1R + WBRT, CD $45^{\text {low/int }} \mathrm{CD} 11 \mathrm{~b}^{+}$) for RNA extraction and RNA sequencing (Fig. 1a).

\section{Microglia depletion and repopulation eliminates radiation-induced transcriptome signatures}

To identify biological pathways involved in radiationinduced memory deficits, we listed genes differentially expressed in microglia after WBRT with and without microglia depletion and repopulation for Gene Ontology Biological Process (GOBP) enrichment analysis. 204 genes were found to be differentially expressed (DE genes) only in microglia isolated from irradiated brains (Fig. 1b and Additional file 2: Table S1). No enriched GOBP terms were found from the 87 WBRT down-regulated genes (Additional file 2: Table S1). There were 193 enriched GOBP terms from the 117 WBRT up-regulated genes, the top 20 enriched GOBP terms are listed in Fig. 1c. Almost half (96) of these enriched GOBP terms were associated with increased response to cell cycle regulation, radiation, DNA repair and stress; the rest enriched GOBP terms were associated with increased metabolism [21], development [12], regulation of protein kinase activity [8], cellular adhesion [4] and other functions (Fig. 1d, Additional file 2: Table S1). Notably, regardless of WBRT, the expression of these WBRTinduced DE genes did not change in cells isolated from brains treated with CSF-1Ri. These results demonstrate that the transcriptomic changes in microglia induced by WBRT can be completely eliminated after microglia depletion and repopulation.

To validate the RNA-seq results, we next performed qPCR analyses using sorted microglia from animals in the same cohort (Fig. 1b, and Additional file 2: Table S1). The expression of the toll-like receptor 3 (TLR3) family gene Lgals 9 was significantly increased by irradiation (WBRT + Control diet versus Sham + Control diet) and was at levels comparable to the shams (Sham + Control diet) when treated with CSF-1Ri despite of irradiation (Additional file 1: Fig. S1a). TNF $\alpha$, another TLR3 family member which also belongs to GOBP "regulation of response to reactive oxygen species (ROS)", was significantly upregulated by irradiation (WBRT + Control diet versus Sham + Control diet); its expression levels are comparable between the Sham + Control diet and the WBRT+CSF1Ri-treated groups. However, TNF $\alpha$ remained elevated in microglia from mice treated only by CSF-1Ri (Additional file 1: Fig. S1b). Another gene from the GOBP "regulation of response to ROS", Sesn2, was also significantly upregulated by WBRT (Additional file 1: Fig. S1c). Sesn2 remained at the control sham levels in CSF-1Ri only group and was significantly downregulated in the WBRT + CSF-1Ri group. $M d m 2$, a gene that belongs to GOBP "cellular response to ionizing radiation", was increased after WBRT, and significantly downregulated in in CSF-1Ri treated groups (Additional file 1: Fig. S1d). Other WBRT-induced expression of radiation-induced genes, Ddias, Rad51, FoxM1 and Check 1, were all at the control sham levels in repopulated microglia regardless of the exposure to WBRT (Additional file 1: Fig. S1e-h). In conclusion, the qPCR validation confirmed that the transcriptomic changes seen in our RNA-seq dataset were reliable. These results suggest that CSF-1Ri mediated microglia depletion during WBRT followed by repopulation eliminated radiation-induced signatures in the microglia transcriptome.

\section{Microglia depletion and repopulation prevents radiation-induced loss of hippocampal PSD-95}

We previously demonstrated that brain irradiation resulted in reduced density of dendritic spines in hippocampal neurons [13]. To accurately determine the effect of WBRT in the intrinsic synaptic protein levels, we measured pre- (Syn1) and post-synaptic (PSD-95) markers in the hippocampus by flow synaptometry [31, 32]. Fractionated hippocampal cell membranes containing synaptosomes were enriched and particles between 1 and $3 \mu \mathrm{m}$ were analyzed to measure synaptic protein levels using mean fluorescent intensities by FACS (Fig. 1e). We observed no changes in pre-synaptic Synapsin 1 protein levels in the hippocampi across all groups (Fig. 1f). However, we measured a significant reduction in post-synaptic protein PSD-95 after WBRT, which was completely prevented by CSF-1R inhibitor mediatedmicroglia depletion (Fig. 1g). These results cement the role of microglia in the radiation-induced loss of postsynaptic components after WBRT.

\section{Repopulated microglia after WBRT originate from peripheral monocytes}

The fractalkine receptor CX3CR1 is expressed in both microglia and peripheral monocytes [33], while chemokine receptor CCR2 is mainly expressed in monocytes [34]. In the $\mathrm{Cx} 3 \mathrm{cr} 1^{\mathrm{GFP} /+} \mathrm{Ccr} 2^{\mathrm{RFP} /+}$ reporter mice, the different expression patterns of GFP and RFP can be used to distinguish microglia (GFP + RFP-) from monocytes $(\mathrm{GFP}+\mathrm{RFP}+)$ [34]. To investigate the cell-of-origin of repopulated microglia in our experimental paradigm we generated bone marrow chimeras with head-protected irradiation using fluorescent labeled bone marrow from $\mathrm{Cx} 3 \mathrm{cr} 1^{\mathrm{GFP} /+} \mathrm{Ccr} 2^{\mathrm{RFP} /+}$ donor mice (Fig. 2a). This allowed partial replacement of bone marrow cells without changing the permeability of the blood-brain barrier 


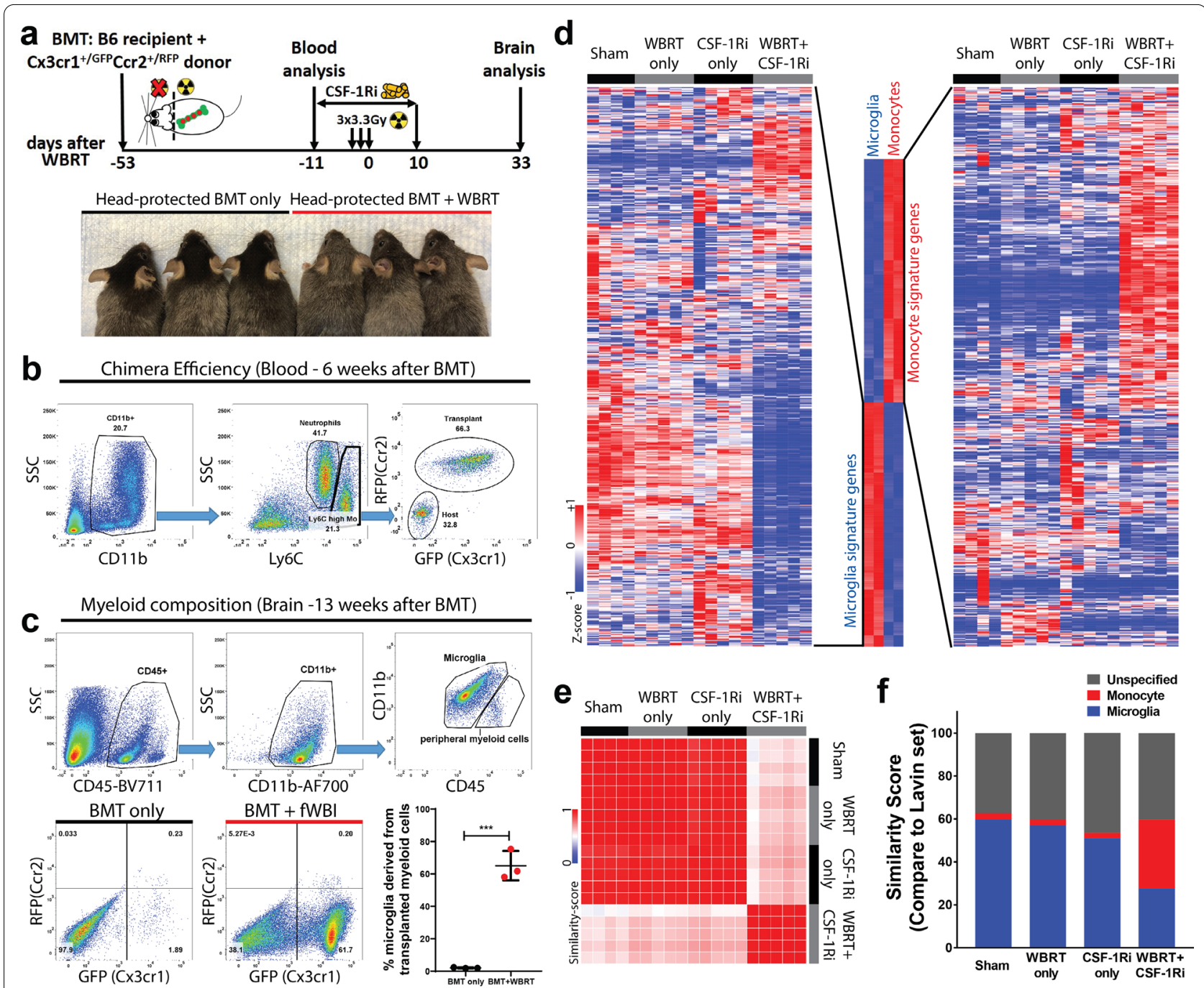

Fig. 2 Repopulated microglia-like cells after depletion and WBRT originate from peripheral monocytes and retain monocytic signatures. a Experimental design of head-protected bone marrow transplantation (BMT) followed by CSF-1Ri-mediated microglia depletion and WBRT. Lower panel shows fur colors before euthanasia for brain analysis. b Representative FACS analysis gating strategy to analyze bone marrow chimera efficiency 6 weeks after BMT, about two-thirds of the CD11 b+ Ly $6 C^{\text {high }}$ monocytes are replaced by GFP+RFP ${ }^{+}$cells derived from donor bone marrow cells. c Representative FACS analysis gating strategy and brain myeloid composition results. Upper panel shows FACS gating using CD45 and CD1 $1 \mathrm{~b}$ staining; microglia and microglia-like cells are defined by positive CD11b staining and low or intermediate CD45 levels. Lower panel shows scatter plots of GFP/RFP fluorescent levels of the $C D 11 b^{+} C D 45$ low/intermediate population in the brain, and a dot plot comparing percentages of peripheral myeloid cell derived microglia-like cells. Statistical analysis was performed using unpaired t-test, ${ }^{* *} p<0.001$. d Hierarchically clustered heatmaps to compare microglia and monocyte signatures. A signature gene list was defined using a dataset published by Lavin and Winter et al., GSE63340. Defined list and expression details are in Additional file 3: Table S2. e Similarity matrix comparisons using defined monocyte and microglia signature genes. $\mathbf{f}$ Bar graph showing similarity scores to compare relative numbers of genes (in percentage of the defined list) that express in the same trends as monocytes or microglia based on the Lavin and Winter et al. dataset

$[2,35,36]$. At 6 weeks after bone marrow transplantation about two-thirds of peripheral monocytes were replaced by transplanted cells with fluorescent labels (Fig. 2b). Bone marrow chimera animals were then treated with WBRT and CSF-1R inhibitor following the same experimental timeline used for RNA sequencing (Fig. 2a). Next, we compared the compositions of myeloid cells in the brain after CSF-1R inhibitor-mediated depletion and repopulation. Flow cytometry analyses performed 33 days after WBRT revealed that microglia depletion and repopulation alone (Sham $+\mathrm{CSF}-1 \mathrm{Ri}$ ) only resulted in limited accumulation of transplanted cells in the brain (Fig. 2c, BMT only). However, in mice that received WBRT and CSF-1R inhibitor two-thirds of the microglia 
were replaced by Cx3cr1-GFP labeled cells, close to the chimera efficiency (Fig. 2b and c, BMT + fWBI). These results suggest that microglia depletion during WBRT resulted in significant contribution of the CNS microglia pool by peripheral monocyte-derived BEMs.

\section{Brain-engrafted macrophages retain monocyte signatures}

We next assessed the transcriptomic profile of the BEMs after microglia depletion and WBRT by comparing our RNA-seq dataset with a previous report by Lavin and Winter et al. [27]. To minimize false discovery and noise signals, we examined 1201 genes from this published dataset with a fold-change greater than 1.50 or smaller than 0.667 for down-regulated genes (FDR $<0.01$, monocyte compared to naïve microglia), and found that 1066 genes were expressed in our samples (Additional file 3: Table S2). Strikingly, the hierarchical clustering of 525 monocyte- and 541 microglia signature genes revealed that the expression profile of monocyte-derived BEMs (WBRT + CSF-1Ri) does not cluster with naive (Sham + Control diet), irradiated (WBRT + Control diet) or repopulated (Sham + CSF-1Ri) microglia (Fig. 2d). Similarity matrix analysis using this microglia/monocyte signature gene list revealed that the expression pattern in BEMs is different from naïve, irradiated and repopulated microglia (Fig. 2e). Next, we counted genes in each group that expressed in the same trends as microglia or monocyte signature genes from the Lavin dataset to determine the similarity scores to these two cell populations. We found that naive, irradiated and repopulated microglia had 60\%, 57\% and 51\% (718, 685 and 612) genes expressed in the same trends as microglia signature genes, respectively, with minimum similarity $(2-3 \%)$ to monocyte signature genes; while BEMs expressed both microglia (28\%, 331 genes) and monocyte signature genes (32\%, 386 genes) (Fig. 2f).

To validate microglia and monocyte signature genes that were differentially expressed in our RNA-seq results we performed qPCR analyses (Additional file 1: Fig. S2 and Additional file 3: Table S2). Microglia signature genes Sall1, P2ry12, Tmem119 and Trem2 were expressed at comparable levels in naïve, irradiated and repopulated microglia, while at significantly lower level in BEMs (Additional file 1: Fig. S2 a-d). On the other hand, expression of monocyte signature gene Runx3, was significantly higher in BEMs than other groups (Additional file 1: Fig. S2e). Notably, previously reported brainengrafted macrophage specific genes Lpar6 and Pmepa1 [37] have significantly higher expression levels in BEMs after CSF-1Ri and WBRT treatments compared to other groups (Additional file 1: Fig. S2f and g). In addition, the expression of $\mathrm{Ccr} 2$, a monocyte signature gene that was not differentially expressed in our RNA-seq dataset, was also not differentially expressed among the four experimental groups by qPCR, suggesting that monocytederived BEMs had lost their Ccr2 expression at this time point (Additional file 1: Fig. S2h). Taken together, these results confirm that after WBRT and CSF-1R inhibitormediated microglia depletion BEMs originate from peripheral monocytes.

\section{Monocyte-derived brain-engrafted macrophages resemble embryonic microglia signatures}

Because monocyte-derived BEMs were exposed to the brain microenvironment for a short period of time, we hypothesized that they were functionally immature. To test this hypothesis, we first examined genes that were highly expressed at different developmental stages in microglia, and used yolk sac/embryonic and adult-specific genes as references (called embryonic and adult signature genes hereon) [28]. Hierarchical clustering of 1617 embryonic and 785 adult microglia signature genes revealed that transcriptomes of BEMs were highly similar to embryonic microglia, while the transcriptomes of microglia from other groups were similar to adult microglia and did not resemble the embryonic microglia (Additional file 1: Fig. S3a, Additional file 4: Table S3). In addition, a similarity matrix analysis using all 2402 overlapped genes between two datasets showed that BEMs had the lowest similarity with microglia from other groups (Additional file 1: Fig. S3b). In addition, 54\% of the listed genes $(n=1306)$ in BEMs expressed in the same trends as yolk sac/embryonic microglia compared to adult microglia (Additional file 1: Fig. S3c). In contrast, naïve (Sham), irradiated (WBRT only) and repopulated microglia (CSF-1Ri only) had much lower embryonic signature similarity scores $(16 \%, 19 \%$ and $17 \%, n=381,445$ and 405, respectively, Additional file 1: Fig. S3c). Notably, naïve, irradiated and repopulated microglia transcriptomes had high adult signature similarity scores (69\%, $59 \%$ and 63\%, $n=1649,1409$ and 1507, respectively), while BEMs had the lowest adult similarity score $(32 \%$, $n=759)$. These data suggest that the monocyte-derived BEMs start to resemble microglia by expressing more embryonic than adult microglia transcriptomic signature genes.

\section{Radiation-induced phagocytosis activity is abrogated in brain-engrafted macrophages}

Aberrant loss of synapses during neuroinflammatory conditions has been linked with increased engulfment of synaptic compartments by microglia [38]. To determine if WBRT affects the phagocytosis potency of microglia, we injected pre-labeled synaptosomes from a naïve donor mouse into the hippocampi of mice after WBRT and CSF-1R inhibitor treatment and measured engulfment 

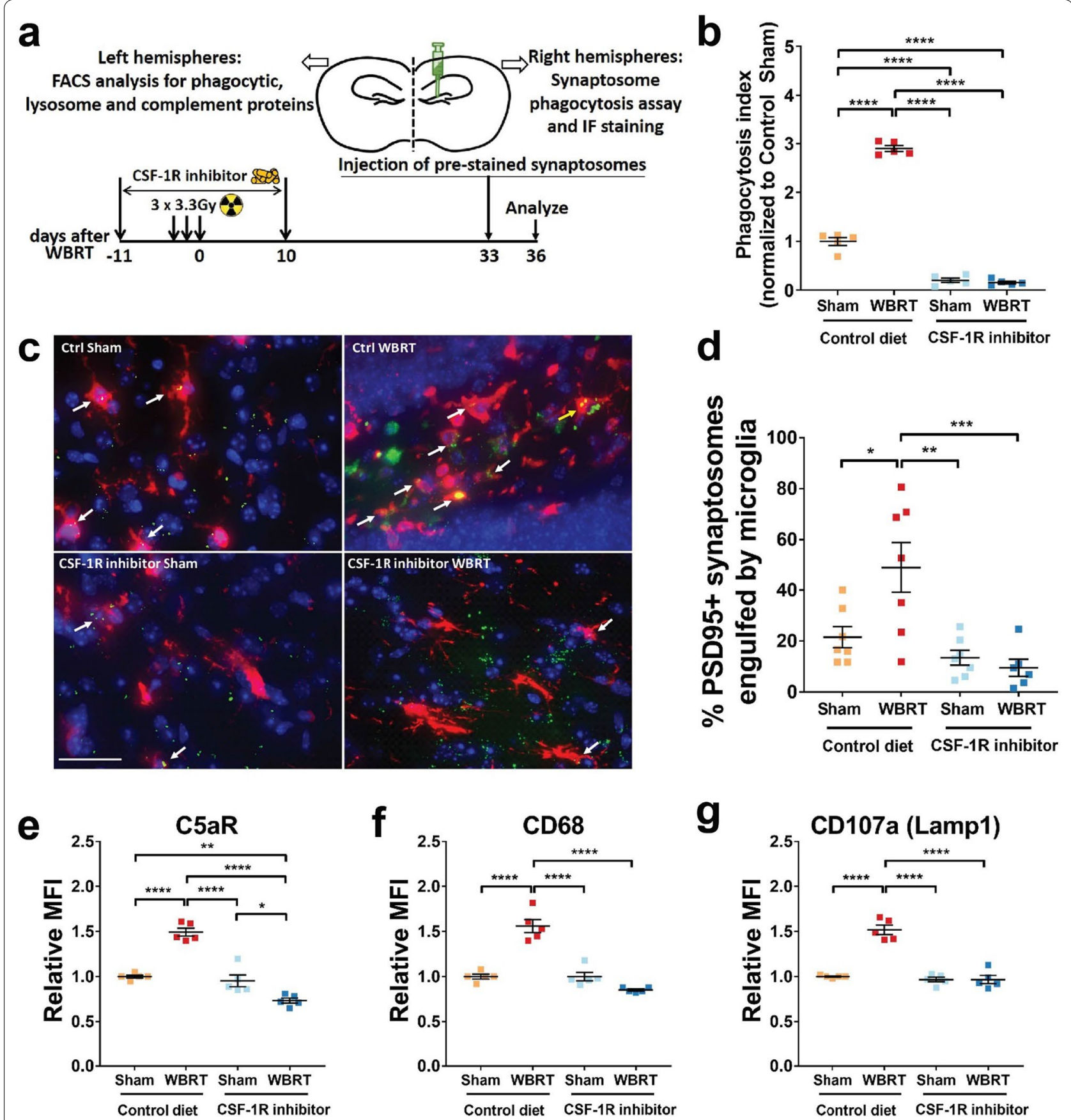

Fig. 3 Repopulated microglia and brain-engrafted macrophages are not activated and phagocyte less synaptic compartments. a Experimental design for in vivo synaptosome phagocytosis assays. Injection of pre-stained synaptosomes was timed to be the same as previous experiments. Three days later, on day 36 after WBRT, ipsilateral hemispheres were harvested and used for engulfment measurement using FACS or

Immunofluorescent staining. b FACS analysis result showing levels of microglia that engulfed pre-stained PSD-95 signals. c Representative images showing engulfment of pre-stained synaptosomes by microglia near injection site. White arrows point at microglia that have engulfed pre-stained synaptosomes. scale bar $=20 \mu \mathrm{m}$. $\mathbf{d}$ Dot plot to show quantification result of synaptosome engulfment by immunofluorescent staining. $\mathbf{e}-\mathbf{g}$ Dot plots showing cell surface C5aR, and intracellular CD68 and CD107a protein levels in microglia and BEMs. Statistical analyses were performed using two-way ANOVA with Tukey's multiple comparisons test. ${ }^{*} p<0.05,{ }^{* *} p<0.01,{ }^{* * *} p<0.001,{ }^{* * * *} p<0.0001$. N $=5-6$ 
by microglia using flow cytometry (Fig. 3a). After WBRT there was a significant increase in the number of microglia engulfing synaptosomes in the hippocampus compared to naïve non-irradiated animals (Fig. 3b). Strikingly, synapse engulfment activity was unchanged compared to naïve animals in animals treated with CSF$1 \mathrm{R}$ inhibitor during WBRT (Fig. 3b). Immunofluorescent imaging at the injection sites confirmed that the injected synaptosomes were indeed engulfed by microglia, and the increased trend of engulfment by irradiated microglia remained unchanged (Figs. $3 \mathrm{c}$ and $\mathrm{d}$, Additional file 1: Fig. S4a). Notably, after hippocampal injection of fluorescent labeled latex beads into the hippocampus, we found that WBRT resulted in increased engulfment of latex beads was also inhibited by CSF-1R inhibitor treatment, suggesting that the WBRT-induced increase of engulfment was not specific to synaptosomes, but rather a general increase of phagocytosis potency (Additional file 1: Fig. S4b). These data are the first to demonstrate that WBRT results in an increase in microglial phagocytosis activity in the hippocampus that can be prevented by transient microglia depletion and full repopulation.

\section{Irradiation-induced complement and phagocytic receptors expression in microglia are absent in BEM after WBRT}

Microglial complement receptors play essential roles in physiologic synaptic elimination during development and aberrant elimination during neuroinflammatory conditions $[38,39]$. To understand the mechanisms of increased microglia phagocytic activity after WBRT, we measured expression levels of a list of complement receptors, phagocytic markers and lysosome proteins in microglia by flow cytometry. The expression of complement receptor $\mathrm{C} 5 \mathrm{aR}$ was significantly elevated in microglia at one month after WBRT. However, in animals treated with CSF-1Ri C5aR expression was unchanged from naive animals (Fig. 3e). The same trend was observed in the expression levels of CD68 and lysosomalassociated membrane protein 1 (LAMP-1) (Fig. 3f and g). These results were consistent with our data demonstrating decreased PSD95 levels (Fig. 3b) and increased microglial phagocytosis activity in the hippocampus after WBRT (Fig. 3b and d). In addition, complement receptor CR3 (CD11b) was significantly elevated in microglia after WBRT or CSF-1Ri treatments alone, and remained unchanged in BEMs with combined WBRT and CSF1Ri treatments (Additional file 1: Fig. S5a). No changes in the complement receptor C3ar1 were measured after WBRT or CSF-1R inhibitor treatment (Additional file 1: Fig. S5b). These results demonstrate that the increased microglia phagocytosis of synaptosomes after WBRT was associated with increased phagocytic and lysosome proteins, and was likely through the complement pathways.

\section{Brain-engrafted macrophages after microglia depletion persist in the brain}

To determine whether BEMs are present long-term in the brain, we monitored this cellular population for 6 months after WBRT. To eliminate the limitation of using bone marrow obtained from the $\mathrm{Cx} 3 \mathrm{cr}^{+/ G F P-}$ Ccr2 ${ }^{+/ R F P}$ knock-in reporter mouse strain, we used an actin-GFP transgenic line as bone marrow donors and generated chimeras using the same body-only irradiation protocol (Fig. 4a). Six weeks later, mice were treated with CSF-1R inhibitor and WBRT and then used to trace BEMs at varies time points (Fig. 4a). Whole coronal sections at the level of the dorsal hippocampus were stained with Iba1 and imaged to quantify total Iba1 + and GFP + cells (Additional file 1: Fig. S6a). We found that all GFP + cells in the brain were also Iba $1+$, suggesting that BEMs were indeed derived from the periphery. In addition, the morphology of Iba1 + GFP + BEMs were analyzed and compared to Iba1+GFP- microglia (Fig. 4b and d). We found that round-shaped Iba1 + GFP + BEM cells entered the brain starting from 7 days after WBRT, and started to obtain more processes that resembled microglia morphology (Fig. 4b). However, Sholl analysis demonstrated that the morphology of BEMs remained stable from 33 days after WBRT and never reached the structural complexity of microglia (Fig. 4c, Additional file 1: Fig. S7). We found that $40-90 \%$ of Iba1 + cells are also GFP + at 14 days after WBRT. This ratio remained at high levels at 1, 3 and 6 months after WBRT (Fig. 4e). Interestingly, although the Iba1+and Iba1+GFP + cell numbers are not fully recovered at 14 days after WBRT, the microglia replacement ratio was similar to the level of later time points (Fig. 5e and Additional file 1: Fig. S6b and c), suggesting a non-competitive manner of brain parenchyma occupancy by microglia and BEMs. These data demonstrate that BEMs enter the brain shortly after WBRT, adapt to a microglia-like morphology and maintain a stable population.

\section{BEMs provide long-term protection against WBRT-induced memory deficits and hippocampal dendritic spine loss}

To measure the long-term cognitive outcomes, we treated a batch of wild-type animals, and tested their recognition memory at 3 and 6 months after WBRT (Fig. 5a). We found that WBRT resulted in persistent loss of recognition memory also at 3 and 6 months, while CSF-1Ri treatment alone did not alter recognition memory performance (Fig. 5b and c). Strikingly, mice that received WBRT along with temporary microglia depletion did not show any memory deficits and 
a BMT w/ head cover

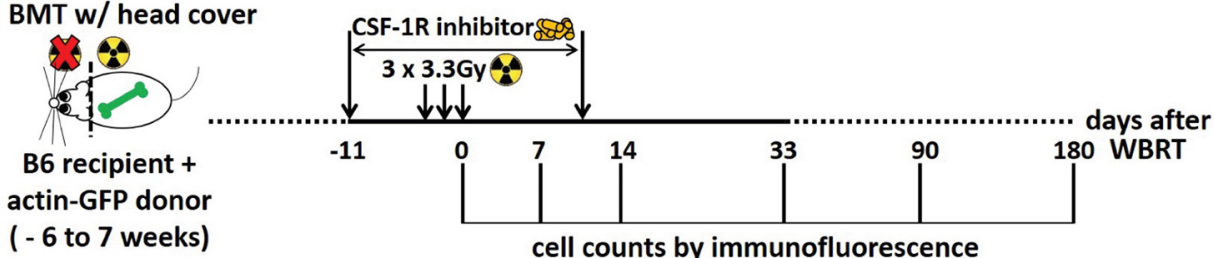

( -6 to 7 weeks)

cell counts by immunofluorescence

b
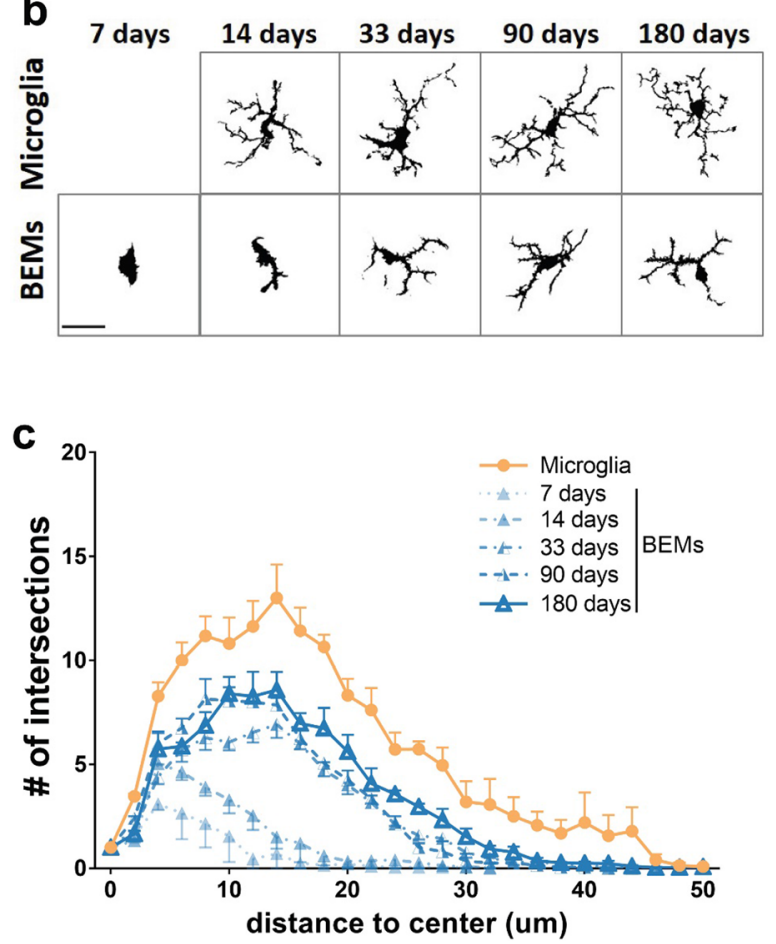

d

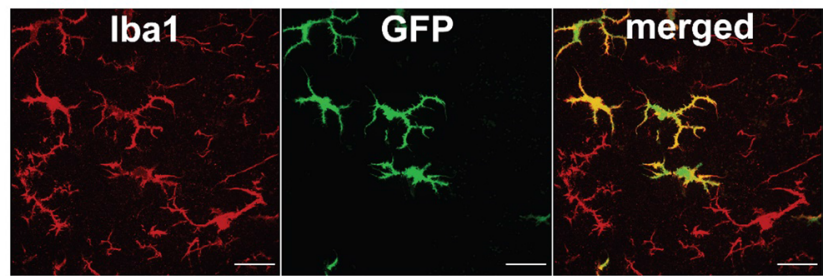

e

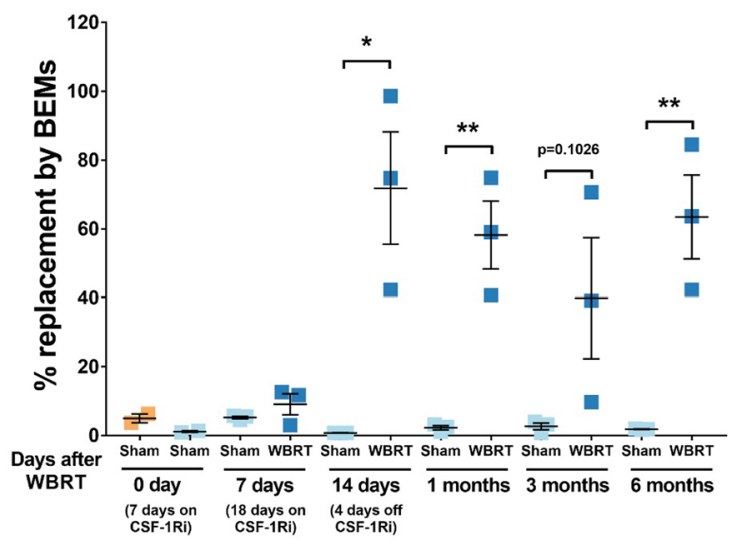

Fig. 4 BEMs gradually adapt to microglia-like morphology and persist in the brain. a Schematic of experimental design for long-term assessment of BEMs. $\mathbf{b}$ Representative images of microglia/BEMs counting, scale bar $=20 \mu \mathrm{m}$. $\mathbf{c}$ Sholl analysis results showing numbers of intersections at different distances to cell center, BEMs at 7, 14, 33, 90 and 180 days after WBRT were compared to naïve microglia age-matched to 90 days after WBRT. d Representative images showing differential Ibal and GFP-expressing profiles of microglia (Iba1 + GFP-) and BEMs (Iba1 + GFP +) in a BEM-bearing brain at 33 days after WBRT. e Dot plot to show percentage of replacement of microglia by BEMs, each dot represent an individual mouse. $n=2-3$. Statistical analyses were performed using unpaired t-test at each distance point (c) or time point (e). See Additional file 1: Fig. S7 for detailed comparisons between microglia and BEMs at each time point

performed undistinguishable from control animals at 3 and 6 months (Fig. 5b and c). Our previous report demonstrated that WBRT-induced dendritic spine loss in hippocampal neurons was fully prevented by temporary microglia depletion during irradiation [13]. In this study, we sought to understand if the protective effects persisted up to 6 months after WBRT. Our results clearly show that radiation-induced loss of dendritic spines in hippocampal neurons persists to this time point, and that the protective effect of microglia depletion and subsequent replacement by BEMs is long lasting (Fig. 5d). Taken together, brief depletion of microglia during WBRT induces sustainable BEMs in the brain and provides long-term protection against irradiationinduced deficits in recognition memory.

\section{Replacement of microglia by BEMs protects against concussive injury-induced memory loss}

To investigate the function of BEMs after they replaced microglia, a single mild concussive $\underline{\text { Closed }} \underline{\text { Head Injury }}$ (CHI) was given to mice 30 days after CSF-1R inhibitor treatment and WBRT; microglia/BEMs morphology and phagocytic activities were measured following recognition memory test by NOR (Fig. 6a). By FACS analysis at 24 days post injury, we found that phagocytosis activity increased $(p=0.0419)$ after $\mathrm{CHI}$ in microglia but not in 

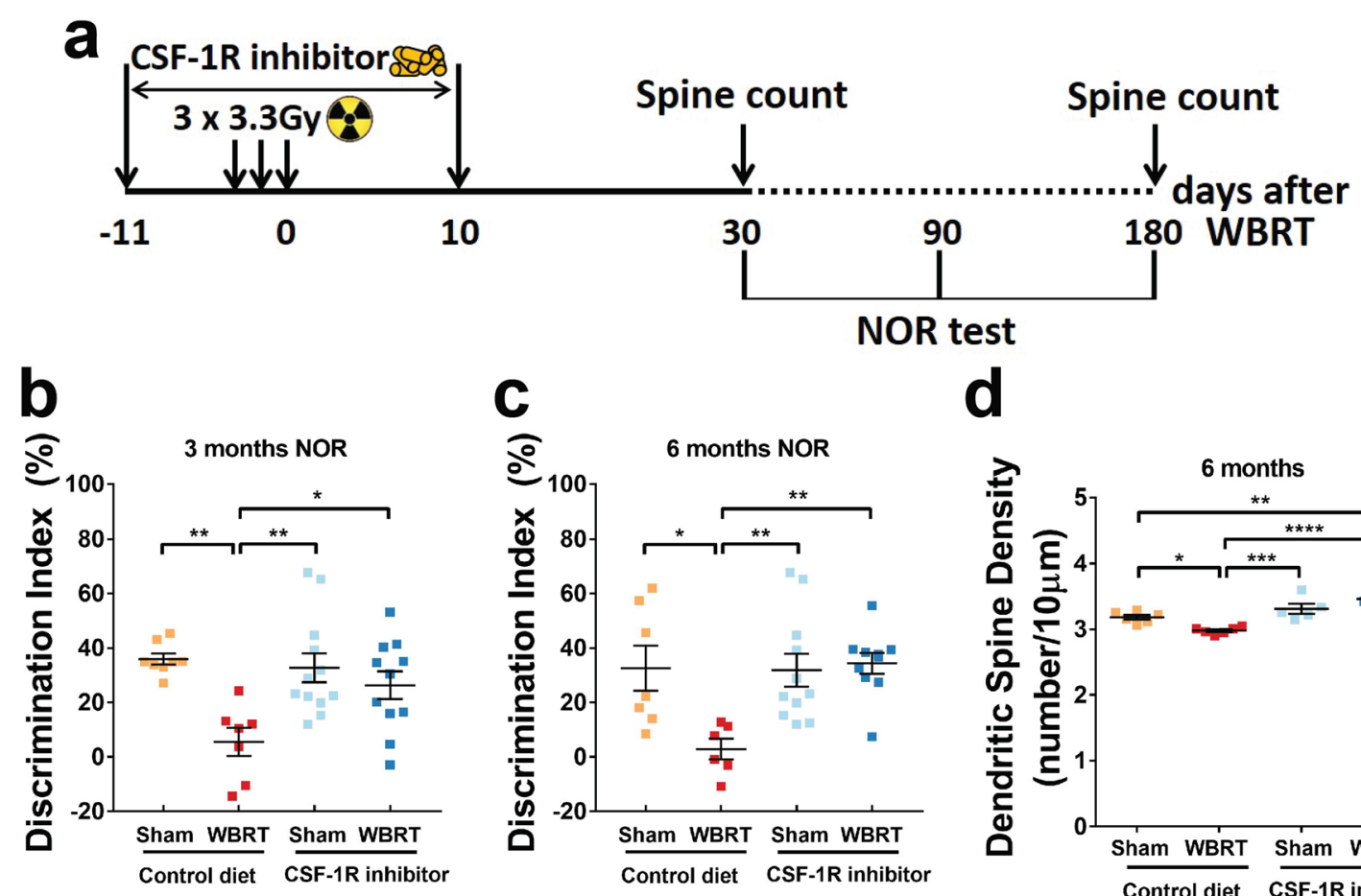

NOR test
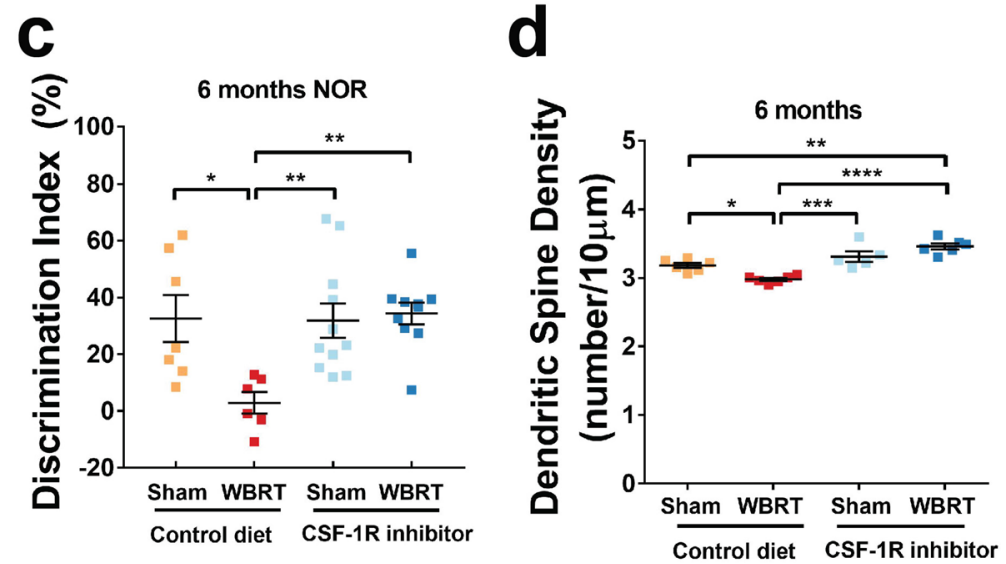

Fig. 5 BEMs provide long-term protection against WBRT-induced dendritic spine and memory loss. a Schematic of experimental design for long-term memory and dendritic spine density analyses. $\mathbf{b}$ and $\mathbf{c}$ dot plots to show NOR test results at 3 and 6 months after WBRT, respectively. $N=6-12$. d Dendritic spine counts of hippocampal granule neurons at 6 months after WBRT, $N=5-6$. Statistical analyses were performed using two-way ANOVA with Tukey's post hoc multiple comparisons test (b-d). ${ }^{*} p<0.05,{ }^{* *} p<0.01,{ }^{* *} p<0.001,{ }^{* * *} p<0.0001$

BEMs (Fig. 6b). Quantification of immunofluorescent staining of Iba-1/PSD-95 co-localization also revealed a trend of increased engulfment towards pre-stained synaptosomes by microglia but not by BEMs (Fig. 6c and d). In addition, the structural complexity of microglia decreased in sholl analysis, while the morphology of BEMs remained unchanged after CHI (Fig. 6e). Furthermore, at 20 days after injury, $\mathrm{CHI}$-induced recognition memory deficits were spared in mice whose microglia were replaced by BEMs (Fig. 6f). These results demonstrate that unlike resident microglia which transition to a less ramified morphology and exhibit increased phagocytic activity towards injected synaptosomes, BEMs remain unchanged in both morphology and phagocytic activity in response to $\mathrm{CHI}$. More importantly, our data suggest that replacement of microglia by BEMs can protect against $\mathrm{CHI}$-induced memory loss.

\section{Discussion}

Here, we provide evidence for the direct involvement of microglia phagocytic activity towards synaptic compartments as a mechanistic cause for loss of dendritic spines with consequent impairments in memory functions after WBRT. Replacement of microglia with monocyte-derived
BEMs prevents loss of synapses and consequent memory deficits. Importantly, BEMs replacing microglia are also protective against a second injury to the brain. Together our results unravel novel immediate and long-lasting therapeutic benefits of microglia depletion and repopulation during WBRT.

Microglia play pivotal roles in reshaping synaptic networks during neonatal brain development [40, 41]. They engulf synaptic elements by active synaptic pruning in an activity- and complement-dependent manner [41]. Microglia-driven aberrant loss of synapses and consequent impairment of cognitive functions have also been reported in animal models of $\mathrm{AD}$ [38], infection [42], injury [43, 44], and aging [45]. Using RNA sequencing, we compared the transcriptomes of microglia from irradiated and non-irradiated brains after CSF-1Ri-mediated microglia depletion and repopulation. WBRT induces increased expression of genes that mainly belong to cell cycle regulation, DNA damage repair and stress-induced biological processes (Fig. 1d). As a result, activated microglia have higher engulfing potential towards both intrinsic and extrinsic synaptic compartments (Figs. 1g, 3b-d). This view is further supported by the increased expression of 


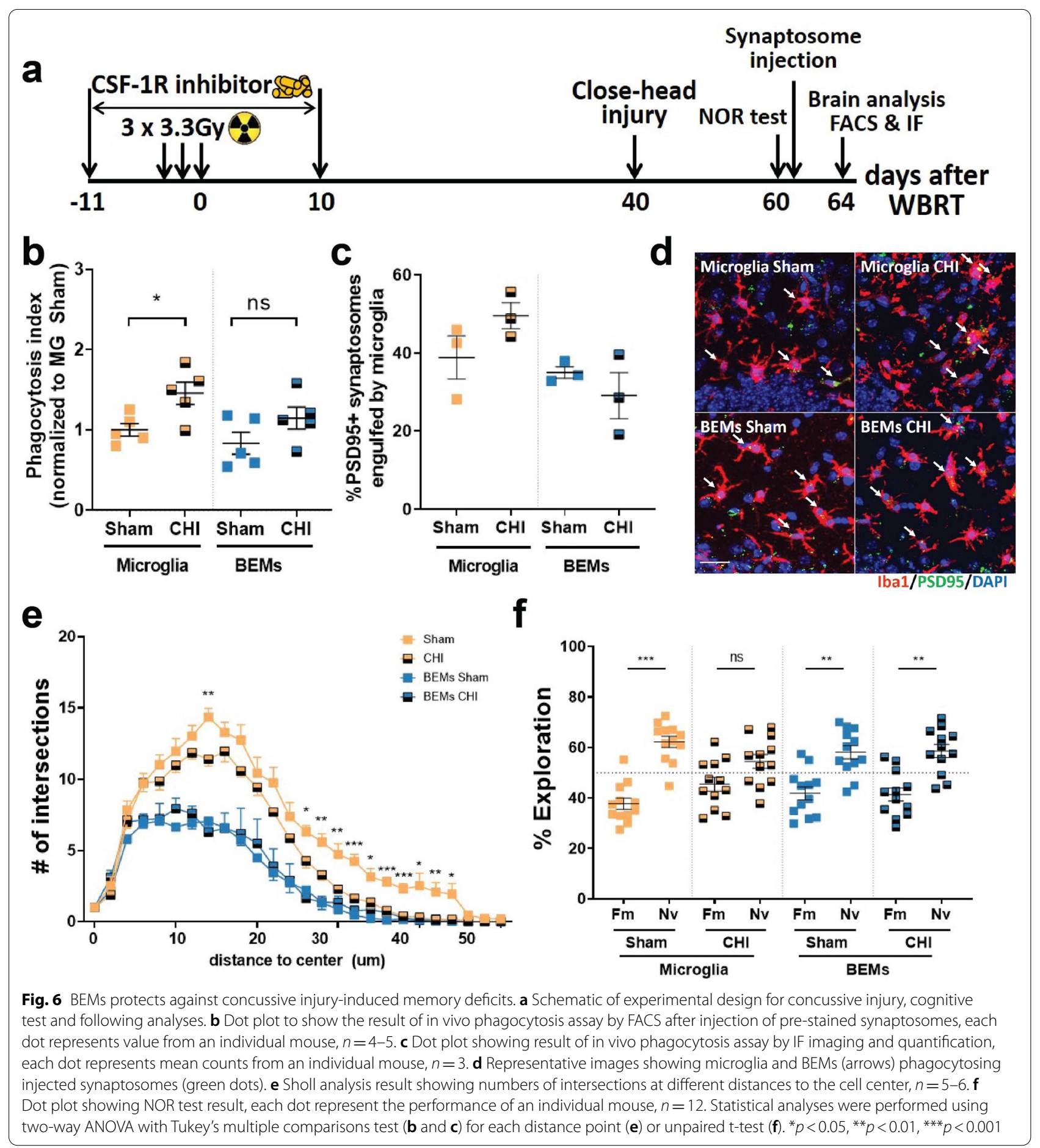

endosome/lysosome proteins CD68 and CD107a with the complement receptors CR3 and C5ar1 measured in microglia chronically after WBRT (Fig. 3e-g, and Additional file 1: Fig. S5). Notably, both endosome/lysosome proteins and complement receptor expressions were comparable to naïve microglia (sham + control diet) in
BEMs (WBRT + CSF-1Ri) and repopulated microglia (CSF-1Ri only). These results suggest that the loss of hippocampal synapses after WBRT may be dependent on the activation of the alternative complement pathway. Interestingly, although BEMs are morphologically similar to adult microglia, they retain a transcriptomic 
signature similar to both circulating monocytes and embryonic microglia (Fig. 2 and Additional file 1: Fig. S3).

The decrease in post-synaptic protein PSD95 level in hippocampal synaptosomes is also paralleled with reductions in hippocampal dendritic spines (Fig. 5d and Feng et al. [13]). However, pre-synaptic Synapsin 1 protein levels are not affected by WBRT or microglia depletion, suggesting that WBRT mainly induces loss of post-synaptic compartments. Interestingly, although the phagocytosis potency of repopulated microglia and BEMs are both low (Fig. 3b and d), microglia depletion and repopulation alone does not affect dendritic spine density (Fig. $5 \mathrm{~d}$ and e). On the other hand, microglia replacement by BEMs results in increased dendritic spine density compared to those with radiation alone, and microglia depletion alone [13]. Strikingly, the protective effect of microglia depletion during WBRT results in protected memory functions and extends to 3 and 6 months following irradiation (Fig. $5 \mathrm{~b}$ and c). The dendritic spine density in mice that received WBRT and CSF-1Ri remained higher than those who only received CSF-1Ri (Fig. 5e) suggesting that in an non-reactivate state (evidenced by no changes in genes involved in cell cycle and radiation response, in microglial phagocytosis and lysosome proteins, and in phagocytosis activity towards injected synaptosomes and latex beads) of repopulated microglia and BEMs may have intrinsic differences in maintaining the homeostasis of dendritic spines, which appears to diminish over time.

In the CNS, microglia maintain a stable population by self-renewal in either a random manner or through clonal expansion [3, 46]. CSF-1R inhibitor treatment alone results in acute depletion of up to $99 \%$ of CNS resident microglia, with repopulated microglia arising solely from the residual microglia and their progenitor cells that remain after treatment $[8,47]$. The repopulated microglia have transcriptional and functional profiles similar to naïve microglia [9]. Peripheral macrophages can engraft into the brain but remain morphologically, transcriptionally and functionally different from CNS resident microglia [48, 49]. Under specific circumstances, monocytes entering the CNS can become microglia-like cells. This is most clearly demonstrated in experiments where lethal whole-body irradiation was followed by bone marrow transplantation with labeled monocytes $\left(\mathrm{Ccr} 2^{+} \mathrm{Ly} 6 \mathrm{C}^{\text {high }}\right)$, resulting in accumulation of these cells in the brain [35]. In neonatal mouse brains monocytes can enter the brain parenchyma without head irradiation and become microglia-like cells at a low frequency [50]. In addition, chronic depletion of microglia without irradiation also results in myeloid cells entering the CNS and becoming BEMs [37]. However, the roles of BEMs in cognitive functions are largely unknown. Here we report that concurrent microglia depletion and therapeutic brain irradiation causes peripheral monocytes to enter the brain parenchyma and become microglia-like BEMs. BEMs enter the brain at 14 days after the completion of brain irradiation, or 4 days after the CSF-1Ri withdrawal (Fig. 4e, and Additional file 1: Fig. S6). Notably, although the ratio of BEMs was high at this time point the total number of Iba1 positive cells is not fully recovered (Additional file 1: Fig. 6b). This ratio remains at high levels in head-irradiated mice throughout the current study (Fig. 5e), suggesting that microglia depletion during WBRT results in sustainable replacement of microglia by BEMs. Importantly, this observation correlates with long-term protection against WBRT-induced loss of recognition memory and dendritic spines in hippocampal granule neurons (Fig. 6b-e).

In the clinic, cancer patients are unlikely to receive a second round of radiotherapy to the brain. Therefore, instead of introducing a second round of WBRT, after they occupied the brain we gave BEM-bearing mice $\mathrm{CHI}$ that causes memory deficits $[51,52]$, and further examined BEMs' response to a single head trauma. Our data show that microglia had increased phagocytic potential to exogenous synaptosomes after $\mathrm{CHI}$, while phagocytic activity of BEMs did not change and remained at a similar level as naïve microglia (Fig. $6 \mathrm{~b}$ and c). This is further demonstrated by Sholl analysis of BEMs showing no change in morphology after CHI (Fig. 6e). Most importantly, $\mathrm{CHI}$-induced memory deficit was prevented in BEM-bearing mice (Fig. 6f). These data are the first to demonstrate that BEMs can prevent brain injury-induced cognitive dysfunction.

A limitation of the current study is that we did not investigate transcriptomic profiles and phagocytic functions of BEMs at chronic time points after they replaced microglia. Therefore, it is unknown whether BEMs can obtain transcriptomic profiles and functions closer to adult microglia at later time points. In addition, microglia have been shown to mediate forgetting through complement-dependent synaptic elimination [53]. We did observe increased dendritic spines in hippocampal granule neurons at both 1 and 6 months after WBRT compared to sham animals, suggesting that BEMs may have lower activity in eliminating synapses than naive microglia. Therefore, the consequences of microglia replacement by BEMs in normal forgetting need further investigation. Furthermore, in the current study we rely solely on the NOR test to measure memory functions. It is possible that the behavioral changes we saw are results from differences in motivation of exploratory behavior or curiosity rather than recognition memory. Additional behavior assays such as Morris Water Maze could be used to detect changes in memory more stringently. Finally, transcriptomic and functional studies at chronic 
time points with other microglia depletion models will provide insights into the transcriptomic and functional dynamics of BEMs in the brain.

Although the CSF-1Ri used in the current study (PLX5622) was only tested in phase I clinical trials, other formats of CSF-1Ri are approved for use in patients. Pexidartinib (PLX3397), which has been shown to deplete microglia with high efficiency $[8,9,11]$, is approved for use in the treatment of symptomatic tenosynovial giant cell tumor [54]. Other CSF-1R Inhibitors including imatinib, dasatinib and bosutinib are approved for use in patients with leukemia and other types of cancer [55]. In addition, a few small-molecule CSF-1R inhibitors are currently under clinical trials. Therefore, the proposed treatment has the potential for quick assessment in clinical phases.

\section{Conclusion}

We report evidence for the mechanism by which microglia depletion and repopulation after WRBT prevents memory loss. Our data demonstrate that replacement of CNS resident microglia by peripheral monocyte-derived BEMs results in a transcriptional and functional reset of immune cells in the brain to an inactive state, which spares the brain from WBRT-induced dendritic spine loss in hippocampal neurons and recognition memory deficits. Most importantly, replacement of microglia by BEMs protects against concussive brain injury-induced cognitive deficits. These results suggest that replacement of depleted microglia pool by peripheral monocyte-derived BEMs represents a potent treatment for irradiation-induced memory deficits.

\section{Abbreviations \\ CNS: Central nervous system; CSF-1R: Colony-stimulating factor 1 receptor; CSF-1Ri: Colony-stimulating factor 1 receptor inhibitor; WBRT: Whole-brain radiotherapy; BEMs: Brain-engrafted macrophages; NOR: Novel Object Rec- ognition; GOBP: Gene ontology biological pathway; DE genes: Differentially expressed genes; FACS: Fluorescence activated cell sorting; $\mathrm{CHI}$ : Closed head injury.}

\section{Supplementary Information}

The online version contains supplementary material available at https://doi. org/10.1186/s12974-021-02290-0.

Additional file 1. Supplementary Figures. Fig S1-S7.

Additional file 2. Table S1. Supporting data for Figure 1.

Additional file 3. Table S2. Supporting data for Figure 2.

Additional file 4. Table S3. Supporting data for Figure S3.

Additional file 5. Table S4. qPCR primers.
Acknowledgements

We thank the Nikon Imaging Center at UCSF for assistance with confocal microscope. We thank the Laboratory for Cell Analysis at UCSF for assistance with FACS sort and analysis.

\section{Authors' contributions}

XF conceptualized the study, designed and performed the experiments, analyzed the data and wrote the manuscript. EF performed experiments, analyzed the data and wrote the manuscript. MP performed experiments, analyzed the data and revised the manuscript. DC assisted in the in vivo phagocytosis assay and data analysis. ZB helped with the in vivo phagocytosis assay imaging and data analysis. MB assisted in the Sholl analysis of microglia and BEMs. SG analyzed the long-term dendritic spine counts data. SL provided assistance in experiments related to WBRT and BM chimeras. NG provided critical inputs to the study and revised the manuscript. SR conceptualized and supervised the study and revised the manuscript. All authors approved the final version of the manuscript.

\section{Funding}

This work was funded by grants from the National Institutes of Health no. R01CA133216, R01CA213441 and R01AG056770 (S.R.).

\section{Availability of data and materials}

The datasets used and analyzed during the current study are available from the corresponding author upon reasonable request.

\section{Declarations}

Ethics approval and consent to participate

All experiments that used animals were conducted in compliance with protocols approved by the Institutional Animal Care and Use Committee at the University of California, San Francisco (UCSF), following the National Institutes of Health Guidelines for Animal Care.

\section{Consent for publication}

Not applicable.

\section{Competing interests}

The authors declare no competing interests.

\section{Author details}

${ }^{1}$ Brain and Spinal Injury Center, University of California San Francisco, San Francisco, USA. ${ }^{2}$ Department of Physical Therapy and Rehabilitation Science, University of California San Francisco, Zuckerberg San Francisco General Hospital, 1001 Potrero Ave, Building 1, Room 101, San Francisco, CA 94110, USA. ${ }^{3}$ Department of Neurological Surgery, University of California San Francisco, San Francisco, USA. ${ }^{4}$ Weill Institute for Neuroscience, University of California San Francisco, San Francisco, USA. ${ }^{5}$ Kavli Institute of Fundamental Neuroscience, University of California San Francisco, San Francisco, USA. ${ }^{6}$ Brain Tumor Research Center, University of California San Francisco, San Francisco, USA. ${ }^{7}$ Department of Pediatrics, University of California San Francisco, San Francisco, USA.

Received: 20 August 2021 Accepted: 6 October 2021

Published online: 15 October 2021

\section{References}

1. Alliot F, Godin I, Pessac B. Microglia derive from progenitors, originating from the yolk sac, and which proliferate in the brain. Dev Brain Res. 1999;117(2):145-52

2. Ginhoux F, et al. Fate mapping analysis reveals that adult microglia derive from primitive macrophages. Science. 2010;330(6005):841.

3. Ajami B, Bennett JL, Krieger C, Tetzlaff W, Rossi FMV. Local self-renewal can sustain CNS microglia maintenance and function throughout adult life. Nat Neurosci. 2007;10:1538.

4. Prinz M, Priller J, Sisodia SS, Ransohoff RM. Heterogeneity of CNS myeloid cells and their roles in neurodegeneration. Nat Neurosci. 2011;14(10):1227-35. 
5. Saijo K, Glass CK. Microglial cell origin and phenotypes in health and disease. Nat Rev Immunol. 2011;11(11):775-87.

6. Hong S, Dissing-Olesen L, Stevens B. New insights on the role of microglia in synaptic pruning in health and disease. Curr Opin Neurobiol. 2015;36:128-34.

7. Hong S, Stevens B. Microglia: phagocytosing to clear, sculpt, and eliminate. Dev Cell. 2016;38(2):126-8.

8. Elmore MR, et al. Colony-stimulating factor 1 receptor signaling is necessary for microglia viability, unmasking a microglia progenitor cell in the adult brain. Neuron. 2014;82(2):380-97.

9. Elmore MR, Lee RJ, West BL, Green KN. Characterizing newly repopulated microglia in the adult mouse: impacts on animal behavior, cell morphology, and neuroinflammation. PLoS ONE. 2015;10(4):e0122912.

10. Dagher NN, et al. Colony-stimulating factor 1 receptor inhibition prevents microglial plaque association and improves cognition in 3xTg-AD mice. J Neuroinflamm. 2015;12(1):139.

11. Spangenberg EE, et al. Eliminating microglia in Alzheimer's mice prevents neuronal loss without modulating amyloid- $\beta$ pathology. Brain. 2016;139:1265.

12. Acharya $M M$, et al. Elimination of microglia improves cognitive function following cranial irradiation. Sci Rep. 2016;6:31545.

13. Feng $X$, et al. Colony-stimulating factor 1 receptor blockade prevents fractionated whole-brain irradiation-induced memory deficits. J Neuroinflamm. 2016;13(1):215.

14. Feng X, Liu S, Chen D, Rosi S, Gupta N. Rescue of cognitive function following fractionated brain irradiation in a novel preclinical glioma model. elife. 2018;7:e38865.

15. Krukowski K, et al. Temporary microglia-depletion after cosmic radiation modifies phagocytic activity and prevents cognitive deficits. Sci Rep. 2018;8(1):7857.

16. Rapp SR, et al. Donepezil for irradiated brain tumor survivors: a phase III randomized placebo-controlled clinical trial. J Clin Oncol. 2015;33(15):1653-9.

17. Stupp R, et al. Radiotherapy plus concomitant and adjuvant temozolomide for glioblastoma. N Engl J Med. 2005;352(10):987-96.

18. Greene-Schloesser D, Robbins ME. Radiation-induced cognitive impairment-from bench to bedside. Neuro Oncol. 2012;14(Suppl 4):iv37-44.

19. Shinohara C, Gobbel GT, Lamborn KR, Tada E, Fike JR. Apoptosis in the subependyma of young adult rats after single and fractionated doses of X-rays. Can Res. 1997;57(13):2694-702.

20. Morganti JM, Jopson TD, Liu S, Gupta N, Rosi S. Cranial irradiation alters the brain's microenvironment and permits CCR2+ macrophage infiltration. PLOS ONE. 2014;9(4):e93650.

21. Warrington JP, et al. Whole brain radiation-induced vascular cognitive impairment: mechanisms and implications. J Vasc Res. 2013;50(6):445-57.

22. Hua K, Schindler MK, McQuail JA, Forbes ME, Riddle DR. Regionally distinct responses of microglia and glial progenitor cells to whole brain irradiation in adult and aging rats. PLOS ONE. 2012;7(12):e52728.

23. Chiang CS, McBride WH, Withers HR. Radiation-induced astrocytic and microglial responses in mouse brain. Radiother Oncol. 1993;29(1):60-8.

24. Mizumatsu S, et al. Extreme sensitivity of adult neurogenesis to low doses of X-irradiation. Can Res. 2003;63(14):4021-7.

25. Monje ML, et al. Impaired human hippocampal neurogenesis after treatment for central nervous system malignancies. Ann Neurol. 2007:62(5):515-20.

26. Mi H, Muruganujan A, Ebert D, Huang $X$, Thomas PD. PANTHER version 14: more genomes, a new PANTHER GO-slim and improvements in enrichment analysis tools. Nucleic Acids Res. 2019;47(D1):D419-26.

27. Lavin Y, et al. Tissue-resident macrophage enhancer landscapes are shaped by the local microenvironment. Cell. 2014;159(6):1312-26.

28. Matcovitch-Natan O, et al. Microglia development follows a stepwise program to regulate brain homeostasis. Science. 2016;353(6301):aad8670.

29. Ferreira TA, Blackman AV. Neuronal morphometry directly from bitmap images. Nat Methods. 2014;11(10):982-4.

30. Schindelin J, et al. Fiji: an open-source platform for biological-image analysis. Nat Methods. 2012;9(7):676-82.

31. Fein JA, et al. Co-localization of amyloid beta and tau pathology in Alzheimer's disease synaptosomes. Am J Pathol. 2008;172(6):1683-92.
32. Prieto $G A$, et al. Synapse-specific IL-1 receptor subunit reconfiguration augments vulnerability to IL-1 $\beta$ in the aged hippocampus. Proc Natl Acad Sci. 2015;112(36):E5078

33. Jung $\mathrm{S}$, et al. Analysis of fractalkine receptor $\mathrm{CX}_{3} \mathrm{CR} 1$ function by targeted deletion and green fluorescent protein reporter gene insertion. Mol Cell Biol. 2000;20(11):4106.

34. Saederup $N$, et al. Selective chemokine receptor usage by central nervous system myeloid cells in CCR2-red fluorescent protein knock-in mice. PLoS ONE. 2010;5(10):e13693.

35. Mildner A, et al. Microglia in the adult brain arise from Ly-6ChiCCR2+ monocytes only under defined host conditions. Nat Neurosci. 2007;10(12):1544-53.

36. Priller J, et al. Targeting gene-modified hematopoietic cells to the central nervous system: use of green fluorescent protein uncovers microglial engraftment. Nat Med. 2001;7(12):1356-61.

37. Cronk JC, et al. Peripherally derived macrophages can engraft the brain independent of irradiation and maintain an identity distinct from microglia. J Exp Med. 2018;215(6):1627-47.

38. Hong $\mathrm{S}$, et al. Complement and microglia mediate early synapse loss in Alzheimer mouse models. Science; 2016.

39. Stevens B, et al. The classical complement cascade mediates CNS synapse elimination. Cell. 2007;131(6):1164-78.

40. Vainchtein ID, et al. Astrocyte-derived interleukin-33 promotes microglial synapse engulfment and neural circuit development. Science. 2018;359(6381):1269.

41. Schafer Dorothy P, et al. Microglia sculpt postnatal neural circuits in an activity and complement-dependent manner. Neuron. 2012;74(4):691-705.

42. Vasek MJ, et al. A complement-microglial axis drives synapse loss during virus-induced memory impairment. Nature. 2016;534(7608):538-43.

43. Norris GT, et al. Neuronal integrity and complement control synaptic material clearance by microglia after CNS injury. J Exp Med. 2018;215:1789.

44. Krukowski K, et al. Traumatic brain injury in aged mice induces chronic microglia activation, synapse loss, and complement-dependent memory deficits. Int J Mol Sci. 2018;19(12):3753.

45. Shi $Q$, et al. Complement C3-deficient mice fail to display age-related hippocampal decline. J Neurosci. 2015;35(38):13029-42.

46. Tay $T L$, et al. A new fate mapping system reveals context-dependent random or clonal expansion of microglia. Nat Neurosci. 2017:20:793.

47. Huang $Y$, et al. Repopulated microglia are solely derived from the proliferation of residual microglia after acute depletion. Nat Neurosci. 2018;21(4):530-40.

48. Ajami B, Bennett JL, Krieger C, McNagny KM, Rossi FMV. Infiltrating monocytes trigger EAE progression, but do not contribute to the resident microglia pool. Nat Neurosci. 2011;14(9):1142-9.

49. Yamasaki R, et al. Differential roles of microglia and monocytes in the inflamed central nervous system. J Exp Med. 2014;211(8):1533-49.

50. Chen $\mathrm{H}-\mathrm{R}$, et al. Fate mapping via CCR2-CreER mice reveals monocyteto-microglia transition in development and neonatal stroke. Sci Adv. 2020;6(35):eaab2119.

51. Chou A, et al. Inhibition of the integrated stress response reverses cognitive deficits after traumatic brain injury. Proc Natl Acad Sci. 2017;114(31):E6420.

52. Krukowski $\mathrm{K}$, et al. Integrated stress response inhibitor reverses sexdependent behavioral and cell-specific deficits after mild repetitive head trauma. J Neurotrauma. 2019;37(11):1370-80.

53. Wang $C$, et al. Microglia mediate forgetting via complement-dependent synaptic elimination. Science. 2020;367(6478):688.

54. Anonymous. CSF-1R inhibitor DCC-3014. NCI drug directory; 2020.

55. Denny WA, Flanagan JU. Small-molecule CSF1R kinase inhibitors; review of patents 2015-present. Expert Opin Ther Pat. 2021:31(2):107-17.

\section{Publisher's Note}

Springer Nature remains neutral with regard to jurisdictional claims in published maps and institutional affiliations. 